\title{
MACROSCOPIC OPTICAL COHERENCE PHENOMENA
}

\author{
E. L. HAHN \\ Physics Dept., University of California, Berkeley, Calif. 94720, USA
}

\begin{abstract}
Principles of coherent radiation are reviewed as they apply to the coherent interaction between two quantum level atomic oscillators and classical radiation fields. The equivalence between electric dipole and magnetic dipole interactions is outlined for macroscopic systems. The power mechanism for formation of coherent signals is briefly calculated. The self-induced transparency mechanism and area-theorem connected with this mechanism serves as a means of predicting properties of photon echoes. It is stressed that when the classical field is a self-consistent solution to both the equations of the density matrix and Maxwell's equations a reaction field need not be added.
\end{abstract}

\section{THE SEMI-CLASSICAL PICTURE}

When the Bloch and Purcell groups initiated the science of $\mathrm{nmr}$, it was not anticipated then or for a number of years afterwards that the dynamics of nmr could be applied to electric dipole resonance transitions. The concepts of spin states dominated the interests of investigators because of the tradition of the Rabi atomic beam method and because radio frequency techniques were ripe for application. Although the gas microwave absorption technique was flourishing, it was not until Dicke ${ }^{1}$ (1954) introduced the idea of radiation coherence from an ensemble of phased electric dipole moments that the classical picture was believed to be applicable to dipole radiation from twolevel systems other than magnetic spins. Dicke pointed out that the coherent superpositions of atomic two-level states could be prepared, after which these states would radiate coherently. In 1957 Feynman, Vernon, and Hellwarth ${ }^{2}$ showed that electric dipole transitions in a two-level system could be formulated by equations which are the analogue of the Bloch torque equations for a spin system. Although the discussion in their paper was restricted to a particular MASER problem, they utilized the viewpoint very well known as the semi-classical method. In this particular discussion the classical electric field is a self-consistent solution of both the 'optical type' Bloch equations, originating from the density matrix, and Maxwell's equations. A number of treatments ${ }^{3}$ and reviews ${ }^{4}$ have appeared with the express purpose of clarifying and justifying the assumptions of the semi-classical approach. The approach has been rather successful from an experimental point of view in predicting and accounting for a number of 


\section{E. L. HAHN}

pulsed laser phenomena seen in recent years, which is the subject of this brief review, such as photon echoes ${ }^{5}$, self-induced transparency ${ }^{6}$, and some properties of mode-locked lasers ${ }^{7}$.

The semi-classical method implies that experimental conditions are restricted to large electric field amplitudes $E(\bar{r}, t)$, and also to large collections of $N$ dipoles. The effective momenta of field and particle oscillators is enormous compared to $\hbar$. The subtle aspects of incoherence due to small quantum fluctuations and spontaneous emission are not easily included in the semi-classical method, and at best is done ad hoc under special conditions. In general, the semi-classical method has not been satisfactorily doctored to include partial damping due to spontaneous incoherent emission except in the usual phenomenological way of introducing damping terms as in the original Bloch equations.

\section{THE TWO-LEVEL SYSTEM}

The equivalence of the $2 \times 2$ transformation Pauli matrices for a twolevel system of spins (each with spin $I=\frac{1}{2}$ ) to that of electron second-quantization operators makes it possible to draw a one-to-one correspondence between ground and excited states of spin states to those of electron states. A torque equation of the form

$$
\frac{\mathrm{d} \bar{P}}{\mathrm{~d} t}=\frac{2 p}{\hbar}\left(\bar{P} \times \bar{E}_{\text {eff }}\right)=\kappa\left(\bar{P} \times \bar{E}_{\text {eff }}\right)
$$

results, where $p$ is the dipole matrix element, $\bar{P}$ is the macroscopic polarization, and $\bar{E}_{\text {eff }}$ is the effective electric field. Equation 1 follows from the density matrix $(\rho)$ method, where the Hamiltonian is written in the dipole approximation as

$$
\begin{aligned}
& \mathscr{H}=\mathscr{H}_{0}-\bar{P}_{\text {op }} \cdot \bar{E}(z, t) \\
& \bar{P}=N \operatorname{Tr}\left\{\rho \bar{P}_{\text {op }}\right\} ;
\end{aligned}
$$

and the energy of the system is

$$
W=N \operatorname{Tr}\left\{\rho \mathscr{H}_{0}\right\}
$$

The ground state energy is

$$
W=W_{0}=-N \frac{\hbar \omega_{0}}{2}
$$

for $N$ dipoles with resonance transition frequency $\omega_{0}$. The correspondence between $\mathrm{nmr}$ and optical dipole resonance superposition states is sketched as follows:

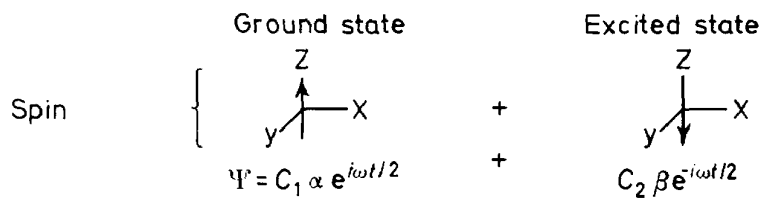




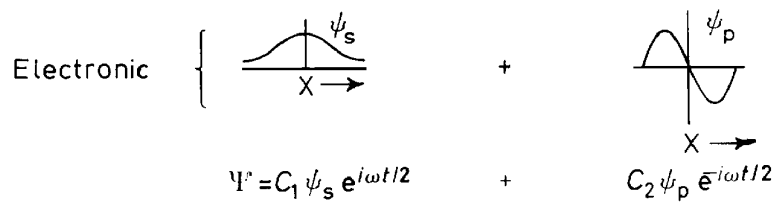

Following a $90^{\circ}$ pulse, the superposition state is prepared at $t=0$ with $C_{1}=C_{2}$, so that a measurement along the $\mathrm{X}$ axis will see:

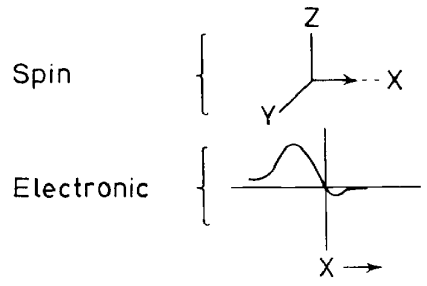

For times $t>0$ the Larmor phase angle $\omega t$ develops, and the precessing spin expectation value appears anywhere in the $x y$ plane. In the electronic case, the electronic wave packet oscillates back and forth between extreme values of $\pm x_{\max }$ at frequency $\omega$.

For an assumed system of electronic $\psi_{\mathrm{s}}$ and $\psi_{\mathrm{p}}$ states, the lack of diagonal electronic dipole moments for $t<0$ in no way detracts from an analogy to the paramagnetic spin case. The magnetization of a spin system is defined as

$$
\bar{M}=\hat{u}_{0} u+\hat{v}_{0} v+\hat{z}_{0} M_{z}
$$

in the frame of reference rotating at frequency $\omega$. In the electronic case it is convenient to define

$$
\bar{P}=\hat{u}_{0} u+\hat{v}_{0} v-\hat{z}_{0} \frac{W \kappa}{\omega_{0}} .
$$

The quantity $W \kappa / \omega_{0}$ appears as a fictitious polarization $-P_{z}$ because of the following correspondence:

$$
P_{z} \equiv M_{z}=-\frac{W \gamma}{\gamma H_{0}}=-\frac{W \kappa}{\omega_{0}} .
$$

Here the energy $W=-M_{z} H_{0}$ of the spin system corresponds to the optical transition total energy $W=N \operatorname{Tr}\left\{\rho H_{0}\right\}$ of the atom where $\left|\mathscr{H}_{0}\right|=\hbar \omega_{0} / 2$ is determined by its structure.

Consider a two-level system tuned at frequency $\omega_{0}$ off-resonance with respect to an existing electric field plane wave

$$
E(z, t)=\mathscr{E}(z, t) \exp \{i[\omega t-k z-\phi(z, t)]\}+c c
$$

propagating in the $Z$ direction at carrier frequency $\omega$. Equation 2 can then be written as

$$
\frac{\partial \bar{P}}{\partial t}=\bar{P} x\left[\hat{u}_{0} \kappa \mathscr{E}(z, t)+\hat{z}_{0}(\Delta \omega+\phi)\right],
$$


where $\Delta \omega=\omega_{0}-\omega$. Using 5,7 may be written out as (with addition of damping terms involving $T_{1}$ and $T_{2}$ )

$$
\begin{aligned}
& \frac{\mathrm{d} u}{\mathrm{~d} t}=v(\Delta \omega+\dot{\phi})-u / T_{2} \\
& \frac{\mathrm{d} v}{\mathrm{~d} t}=-u(\Delta \omega+\dot{\phi})-\frac{K^{2}}{\omega} \mathscr{E} W-v / T_{2} \\
& \frac{\mathrm{d} W}{\mathrm{~d} t}=v \mathscr{E} \omega-\frac{W-W_{0}}{T_{1}} .
\end{aligned}
$$

The Bloch type equations 8 include a possible frequency modulation term $\dot{\phi}(z, t)$, which together with $\mathscr{E}(z, t)$ (the slowly varying envelope), are terms to be self-consistent with equation 7 and Maxwell's equation:

$$
\frac{\partial^{2} E(z, t)}{\partial z^{2}}=\left[\frac{\eta^{2}}{c^{2}} \frac{\partial^{2} E(z, t)}{\partial t^{2}}+\frac{4 \pi}{c^{2}} \frac{\partial^{2} P(z . t)}{\partial t^{2}}\right]
$$

In the slowly varying wave approximation,

$$
\frac{\partial \mathscr{E}}{\partial t} \ll \frac{\mathscr{E}}{i} ; \frac{\partial \mathscr{E}}{\partial t} \ll \omega \mathscr{E}
$$

is assumed. Combining equations 8 with 9 gives, therefore,

and

$$
\frac{\partial \mathscr{E}(z, t)}{\partial z}=\frac{-2 \pi \omega}{\eta c} \int_{-\infty}^{\infty} v g(\Delta \omega) \mathrm{d} \Delta \omega
$$

$$
\mathscr{E} \frac{\partial \phi}{\mathrm{d} z}(z, t)=\frac{2 \pi \omega}{\eta c} \int_{-\infty}^{\infty} u g(\Delta \omega) \mathrm{d} \Delta \omega .
$$

A retarded time frame of reference is used, where the retarded time is given by $t^{\prime}=t-\eta z / c$. Equations 10 and 11 together with 8 are useful for the analysis of pulse propagation at or near resonance in a two level system. These equations can be generalized to cases where the two-level system is degenerate $^{6,7}$, and not necessarily characterized by one dipole matrix element. In this case $v$ and $u$ are replaced by sums $\sum_{i} v_{i}, \sum_{i} u_{i}$ corresponding
to sums over $i$ matrix elements.

If the slowly varying wave approximation is not made, and equation 9 is applied with no approximations, then terms of the type $\omega \dot{v}, \omega \dot{u}, \ddot{v}, \ddot{u}$ are included also in equations 10 and 11 (along with $\omega^{2} v$ and $\omega^{2} u$ which dominate). It is easily seen ${ }^{8}$, however, that the size of these higher order terms is of the same order of magnitude as the off-resonance response contribution by dipole matrix elements in the atom involving transitions to quantum levels other than the two-level system at or on resonance. There is no such thing as a pure two-level optically responsive atom. Other levels must contribute, 


\section{MACROSCOPIC OPTICAL COHERENCE PHENOMENA}

as is dictated by the sum rule for optical response. Unless other optical levels are taken into account, it is quite possible to arrive at unrealistic conclusions concerning optical pulse propagation effects if equation 9 is coupled exactly to equation 8 , which pertains strictly only to a two-level system.

\section{RADIATION DAMPING}

The self-consistent field $\overline{\mathscr{E}}$ in the previous discussion is connected with a source term $\bar{P}$ such that stimulated emission and absorption will be naturally accounted for during propagation in the medium, depending upon the initial conditions with regard to $\bar{P}$ and $\overline{\mathscr{E}}$. If $\overline{\mathscr{E}}_{0}$ is initially thought of as an applied field, then a reaction field originating from $\bar{P}$ will add or subtract to $\overline{\mathscr{E}}_{0}$. The total $\overline{\mathscr{E}}$ however naturally includes any reaction fields, and no extra ones need be added to account for effects due to $\bar{P}$. The self-consistent relationship between $\bar{P}$ and $\overline{\mathscr{E}}$ will automatically include the effects of coherent radiation damping. This is strictly the case for a plane wave propagating through an infinite medium of resonant dipoles. Interference and loss, because of diffraction edge effects due to sample size and finite beam profile, will of course enter the picture if $\bar{P}$ is confined to a finite volume.

If radiation energy is lost from a finite volume element of dipoles, and not recovered by the resonance capture and re-emission of energy in another volume element, it is convenient to introduce what appear to be loss or 'coherent radiating damping' terms'. Such terms are convenient in cases where the dipole system is first prepared by strong field pulses (considered hardly affected by the medium) in coherent superposition states. After the system is prepared it radiates a coherent field by itself, namely, a spontaneous but coherent $\mathscr{E}$ field. The photon echo is a clear cut example of this ${ }^{5}$.

\section{DAMPING OF SPINS IN A CAVITY}

In an external magnetic field $\bar{H}_{0}$, the spin energy of a magnetic moment $M_{0}$ tipped from the field $\bar{H}_{0}$ direction by an angle $\varphi$ is given by

$$
W_{\mathrm{spin}}=-\xi V_{\mathrm{c}} M_{0} H_{0} \cos \varphi .
$$

The $z$ component of magnetization is $M_{z}=M_{0} \cos \varphi, \xi$ is the sample filling factor, and $V_{c}$ is the cavity volume. With the voltage of nuclear induction given by

$$
\mathscr{V}=4 \pi N A \xi \omega M_{0} \sin \varphi,
$$

and $v=M_{0} \sin \varphi$ the energy conservation condition equation 9 requires that

$$
\frac{\mathrm{d} W_{\text {cavity }}}{\mathrm{d} t}=-\frac{\mathrm{d} W_{\text {spin }}}{\mathrm{d} t}=\frac{\mathscr{V}^{2}}{2 R}
$$

in an LCR circuit tuned to nuclear resonance at $\gamma H_{0}=\omega_{0}$. After a super- 


\section{E. L. HAHN}

position state has been established by a $\theta_{0}$ pulse at $t=t_{0}$ it follows from equations 12,13 and 14 that the free procession of field generated by the spins is

$$
H_{1}(t)=\frac{1}{\gamma \tau_{\mathrm{r}}} \operatorname{sech}\left[\left(t+t_{\mathrm{o}}\right) / \tau_{\mathrm{r}}\right]
$$

The radiation damping rate is expressed by

$$
\frac{1}{\tau_{\mathrm{r}}}=2 \pi M_{0} \gamma Q \xi
$$

with $Q=\omega L / R$, assuming $Q / \omega \ll \gamma H_{1}$, and no inhomogeneous broadening is present. For $\theta_{0}=90^{\circ}$ the free nuclear precession signal power is

$$
P_{\max }=8 \pi \xi^{2} Q M_{0}^{2} \omega V_{\mathrm{c}} \approx \frac{M_{0} H_{0}}{\tau_{r}} V_{\mathrm{s}}
$$

where $\xi=V_{\mathrm{s}} / V_{\mathrm{c}}$, and $V_{\mathrm{s}}$ is the sample volume.

\section{Effect of inhomogeneous broadening on the radiation damping}

The equation for an $L C R$ cavity coupled at resonance with the spins is

$$
\frac{\mathrm{d} \omega_{1}}{\mathrm{~d} t}+\frac{R}{2 L} \omega_{1}=\frac{-\omega \gamma \pi V_{\mathrm{s}}}{V_{c}} \int_{-\infty}^{\infty} v(\Delta \omega, t) g(\Delta \omega) \mathrm{d} \Delta \omega,
$$

where $\omega_{1}=\gamma H_{1}$, and a homogeneous field distribution is given by $g(\Delta \omega)$, normalized so that $\int_{-\infty}^{\infty} g(\Delta \omega) \mathrm{d} \Delta \omega=1$. For

equation 17 is written as

$$
\frac{R}{L} \omega_{1} \gg \mathrm{d} \omega_{1} / \mathrm{d} t
$$

$$
\frac{\mathrm{d} \varphi}{\mathrm{d} t}=-\frac{1}{M_{0} \tau_{r}} \int_{-\infty}^{\infty} g(\Delta \omega) v(\Delta \omega, t) \mathrm{d} \Delta \omega .
$$

If $\varphi=\theta_{0}$ at $t=t_{0}$, equation 17 yields, after some manipulation ${ }^{10}$,

or

$$
\Delta \theta=\phi(\infty)-\theta_{0}=-\frac{1}{M_{0} \tau_{r}} v(0, t=\infty) \pi g(0)
$$

$$
\Delta \theta=-\frac{\pi}{\tau_{r}} g(0) \sin \left(\theta_{0}+\theta_{f}\right),
$$

where $\theta_{\mathrm{f}}=\phi(\infty)$ is the final tipping angle at $t=+\infty$. Suppose $\theta_{0}=\pi / 2$. Then

$$
-\frac{T_{2}^{*}}{\tau_{R}} \cos \Delta \theta \approx-\frac{T_{2}^{*}}{\tau_{R}}=\Delta \theta, \text { for } \Delta \theta \ll 1 \text { and } T_{2}^{*} / \tau_{r} \ll 1
$$

The free precession signal lasts for a time $T_{2}^{*}$, during which time the energy 


\section{MACROSCOPIC OPTICAL COHERENCE PHENOMENA}

$\Delta W=-M_{0}\left(\cos \theta_{\mathrm{f}}-\cos \theta_{0}\right) H_{0} \approx-M_{0} H_{0} \theta_{0} \Delta \theta$ is given up to the cavity as signal energy. The free precession signal power is therefore

$$
\frac{\mathrm{d} W}{\mathrm{~d} t} \approx P_{\max } \approx \frac{-M_{0} H_{0} \theta_{0} \Delta \theta}{T_{2}} \approx \frac{M_{0} H_{0}}{\tau_{r}}
$$

near $t=0$, just after a $\pi / 2$ pulse (for $\theta_{0}=\pi / 2$ ), the same power of free precession as given by equation 16 , where inhomogeneous broadening is absent. The results of equations 16 and 20 show as an example that the signal power extracted by the cavity from the coherent system is the same whether or not inhomogeneous broadening is present. At any time $t, M_{0}$ is reduced by inhomogeneous broadening, but also by damping time constants $T_{2}, T_{1}$ which may be shorter or longer than $\tau_{r}$. It is this power expressed by equation 20 which makes possible the observation of various types of echo and free precession signals.

\section{Coupling to the cavity with no resistive losses}

For $R / L \rightarrow 0$ in equation 17 , and $T_{2}^{*}=\infty$, then

$$
\frac{\mathrm{d}^{2} \varphi}{\mathrm{d} t^{2}}=-N p \pi \gamma \omega_{0} \xi \sin \varphi=\frac{-1}{\tau_{\mathrm{c}}^{2}} \sin \varphi .
$$

A number of pendulum solutions are assigned to this equation. The finite single pulse solution expresses the spontaneously developed rf field as

$$
H_{1}(t)=\frac{2}{\gamma \tau_{\mathrm{c}}} \sin \varphi / 2=\frac{2}{\gamma \tau_{\mathrm{c}}} \operatorname{sech} t / \tau_{\mathrm{c}}
$$

if $M_{0}$ is initially inverted at $t=-\infty$ and then tipped infinitesimally to get it to radiate into the cavity. The maximum spin energy is $\Delta W_{\max }=V_{\mathrm{s}} M_{0} H_{0}$ $(\cos \varphi-1)$; and the maximum cavity energy is $\left(2 H_{1}\right)^{2} V_{\mathrm{s}} / 8 \pi \stackrel{\max }{=} W_{\mathrm{c}}$. It is seen from above that $W_{\mathrm{c}} / \Delta W_{\max }=1$. The time constant $\tau_{\mathrm{c}}$ is a characteristic coherence or cooperation time serving to define a coherence volume relevant to discussions concerning coherent propagation and radiation ${ }^{11}$.

\section{RESONANCE INTERACTION OF PROPAGATING PULSES}

The self consistent solution of equations 8 and 9 yields the distance dependence for the pulse area

$$
\theta(z)=\kappa \int_{-\infty}^{\infty} \mathscr{E}(z, t) \mathrm{d} t
$$

to be

$$
\frac{\mathrm{d} \theta}{\mathrm{d} Z}= \pm \frac{\alpha}{2} \sin \theta
$$

For an absorber the minus sign applies, and for an amplifier the plus sign applies. Equation 21 is valid in the slow wave approximation, where frequency modulation is neglected $(\dot{\phi}=0)$, and the applied frequency $\omega$ is set at the centre of a symmetric $g(\Delta \omega)$ distribution. The pulse width $\tau$ may be greater 


\section{E. L. HAHN}

or less than $g(0) \sim T_{2}^{*}$. For an absorber all input areas evolve toward zero, $2 \pi$, or multiples thereof; and for an amplifier, the areas evolve unstably toward $\pi$ or odd multiples thereof. The angle $\theta$ measures the quantum mechanical superposition of states at exact resonance $(\Delta \omega=0)$ imposed by the pulse (after $t=+\infty$ ). The superposition is given by

$$
\psi=\psi_{1} \cos \theta / 2+i \psi_{2} \sin \theta / 2 \text {. }
$$

Equation 21 can be interpreted as expressing the evolution of the Fourier amplitude of pulse at exact resonance in the interaction representation (or rotating frame). Thus, if we define the Fourier transform as

$$
\tilde{\mathscr{E}}(z)=\theta / \kappa=\int_{-\infty}^{\infty} \mathscr{E}(z, t) \exp \left(i \omega t-\omega_{0} t\right) \mathrm{d} t \text { with } \omega-\omega_{0}=0 .
$$

a spectrograph will measure the spectral power density function

$$
I=\frac{\mathrm{c}}{4 \pi} \frac{\theta^{2}}{\kappa^{2}}=I(0) n^{2}
$$

where $I(0)=(c / 4 \pi) \mathscr{E}_{\max }^{2} \tau^{2}$, and $\theta=n 2 \pi$ (for an absorber). For any inhomogeneously broadened two-level system, with degeneracies, one may generally write

$$
\frac{\mathrm{d} \theta}{\mathrm{d} z}(\dot{z})=\mp \frac{\alpha}{2} S(\theta, z)-\sigma / 2 \theta ;
$$

and

$$
\frac{\mathrm{d} \mathscr{J}(z)}{\mathrm{d} z}=\mp \alpha \mathscr{J}(z) F(\theta, z)-\sigma \mathscr{J}(z),
$$

The pulse energy is defined by $\mathscr{J}=\eta_{\mathrm{c}} / 4 \pi \int_{-\infty}^{\infty} \mathscr{E}\left(z^{2}, t\right) \mathrm{d} t ; \sigma$ is a scattering loss,

and $S$ and $F$ are non-linear functions. For a non-degenerate two-level system, $S=\sin \theta$. For a Gaussian pulse with $\tau \gg T_{2}^{*}$ it is reasonably (but empirically) accurate to write $F \cong 2(1-\cos \theta) / \theta^{2}$. The minus sign applies for an absorber, and the plus sign for an amplifier.

In the classical regime $S \approx \theta$, and $F=1$. In the regime of self-induced transparency, $S=\sin \theta, \quad \theta \rightarrow n z \pi$, the electric field $\mathscr{E}=2 / \kappa \tau \times$ $\operatorname{sech}[(t-z / V) / \tau]$, and $F \rightarrow 0$.

In general (for an absorber)

$$
\theta(z)=2 \tan ^{-1}\left\{\exp \left(-\alpha z / z^{\prime}\right) \tan \theta_{0} / 2\right\}
$$

and

$$
\mathscr{J}(z) \approx \mathscr{J}(0) \exp \left[-\alpha \int_{0}^{z} \mathrm{~d} z^{\prime} F\left(0, z^{\prime}\right)\right]
$$

The self-induced transparency condition is determined by a process in which energy absorbed from the pulse by the two-level system during the first half of the pulse is returned to the pulse by stimulated coherent emission during the second half of the pulse. The number of photons brought into 
the volume element of dipoles, in pulse time $\tau$, is equal to the number of dipoles excited.

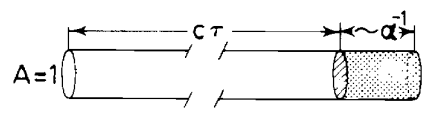

The number of photons is

$$
\mathcal{N} \equiv A \frac{\eta \mathscr{E}^{2} c \tau}{4 \pi \hbar \omega}=A N \frac{T_{2}^{*}}{\tau} \alpha^{-1},
$$

where $T_{2}^{*} / \tau$ is the fraction of atoms excited by the Fourier components of the pulse $(1 / \tau)$ out of a much broader spectrum $\left(1 / T_{2}^{*}\right)$ of inhomogeneously broadened two-level absorbers. The active dipoles occur over a volume $=$ length $\alpha^{-1} \times$ unit area $A(=1)$. Therefore

$$
\frac{2 p^{2}}{\hbar^{2}} \mathscr{E}^{2} \tau^{2}=1, \quad \text { or } \theta \approx \kappa \mathscr{E} \tau=\mathrm{constant}
$$

Careful use of the equations gives the constant area condition $\theta=2 \pi$.

Another useful condition which signifies the existence of a stable $2 \pi$ hyperbolic secant propagating pulse is the fact that it is slowed down to an inverse pulse velocity

$$
\frac{1}{V_{T}}=\eta / c+\frac{1}{V} \text {, and it is possible to have } V \ll c,
$$

so that the medium stores much of the original pulse energy ${ }^{6}$. The expression

$$
\frac{\mathrm{d} \mathscr{J}}{\mathrm{d} z}=-\Delta W \approx \frac{\Delta \mathscr{J}}{\Delta z}=\frac{\eta c}{4 \pi} \frac{\mathscr{E}_{0}^{2} \tau}{\alpha^{-1}}
$$

leads to the ratio ${ }^{12}$ of the field energy to the dipole energy in the same volume to be

$$
\frac{\eta \xi_{0}^{2} / 4 \pi}{\Delta W}=\frac{1}{c \alpha \tau} \sim \frac{V}{c}
$$

where self-induced transparency theory gives $1 / V \sim \alpha \tau$. For no absorbers present we note that $V=\infty$

\section{SELF-INDUCED TRANSPARENCY AND THE PHOTON ECHO}

The photon echo ${ }^{5}$ was first obtained and conceived in terms of the response of a small slab of dipole radiators $\left(\mathrm{Cr}^{3+}\right.$ in $\left.\mathrm{Al}_{2} \mathrm{O}_{3}\right)$ following excitation by a pair of $\mathscr{E}$ field pulses separated by a time $T$. The superposition of two-level optical states results in a spontaneous echo at $t=2 T$.

The spontaneous emission rate in $\operatorname{erg~s}^{-1}$ for a single dipole $p_{0}$ is given by

$$
\dot{W}_{\mathrm{rad}}=\frac{64 \pi^{4} p_{0}^{2} \eta}{3 \lambda^{3} h}(\hbar \omega)
$$




\section{E. L. HAHN}

If out of $N$ dipoles per $\mathrm{cm}^{3}$, an effective macroscopic moment $\left(N p_{0}\right)_{\text {eff }}$ is radiating, then $\left(N p_{0}\right)_{\text {eff }}$ replaces $p_{0}$ in equation 24 , and $\dot{W}_{\text {rad }} \propto N^{2}$. In carrying out the problem this way, special pains must be taken to introduce a coherence volume $\left(\sim \lambda^{2} l\right)$ over which the dipoles cooperate while radiating in the forward direction. The result ${ }^{5}$ for the number of photons in the echo is

$$
\mathscr{N}=\frac{\eta c}{4 \pi} \frac{\mathscr{E}^{2} \tau}{\hbar \omega}=\frac{l\left|\dot{W}_{\mathrm{rad}}\right|}{\hbar \omega} \propto \frac{N^{2} T_{2}^{* 2} l^{2} p_{0}^{2}}{\hbar \lambda \tau}
$$

for a sample of unit area cross-section and small thickness $l<\alpha^{-1}$.

The area theorem equation 21 of self-induced transparency yields the expression for $\mathcal{N}$ directly. For two pulses which do not overlap, it has been shown ${ }^{6}$ for small sample lengths $l=z$ that the echo area is given by

$$
\kappa \mathscr{E}_{0} \tau=\theta_{\mathrm{E}} \approx \frac{1}{2} \alpha l \sin \theta_{1}(0)\left[1-\cos \theta_{2}(0)\right],
$$

an expression similar to that for magnetic spin-echoes, where $\theta_{1}(0)$ and $\theta_{2}(0)$ are the input pulse areas at $z=0$, with pulse width $\tau \ll T$, and $T$ is the pulse separation. The energy in the echo is proportional to $\theta_{\mathrm{E}}^{2} / \tau$, so that

$$
\frac{\eta c}{4 \pi} \mathscr{E}_{0}^{2} \tau=\frac{\eta c}{4 \pi} \frac{\theta_{\mathrm{E}}^{2}}{k^{2} \tau}=W \text {, and } \theta \approx \alpha l .
$$

The number of photons in the echo is therefore

$$
\mathcal{N} \approx \frac{W}{\hbar \omega}=\frac{\eta c}{4 \pi} \frac{1}{\kappa^{2} \tau}(\alpha l)^{2} \sim \frac{N^{2} T_{2}^{* 2} l^{2} p_{0}^{2}}{\hbar \lambda \tau},
$$

which is the same result obtained in equation 25 . The self-consistent application of Maxwell's equations yields the photon echo in a natural way by taking into account the cooperation volume automatically in the plane wave problem.

Campaan and Abella ${ }^{13}$ observe that the intensity $I$ of photon echoes from ruby samples with various concentrations $N$ of $\mathrm{Cr}^{3+}$ does not obey the expected coherent radiation dependence $I \alpha N^{2}$ as $N$ is increased beyond $N \sim 2 \times 10^{-2}$ per cent by weight. They attribute the anomaly to radiation damping corrections, yet to be worked out. However, application of the area theorem as it stands is capable of accounting ${ }^{14}$ for their results. As stated before, the photon echo is a natural consequence of the area theorem for an absorber

$$
\frac{\mathrm{d} \theta}{\mathrm{d} z}=-\frac{\alpha}{2} \sin \theta,
$$

where $\theta=\theta_{1}(z)+\theta_{2}(z)+\theta_{\mathrm{E}}(z)$, and $\alpha$ is the Beer absorption coefficient proportional to $N$. If two pulses with areas $\theta_{1}(0)$ and $\theta_{2}(0)$ are applied to the sample at $z=0$, a third and possibly more pulses with area

$$
\theta_{\mathrm{E}} \cong \sum_{i} \kappa \mathscr{E}_{\mathbf{E} i} \tau_{\mathbf{E} i}
$$

will appear subsequently, in order that the total $\theta$ can evolve either toward a multiple of $2 \pi$ or 0 . This results because multiple echoes which make up 


\section{MACROSCOPIC OPTICAL COHERENCE PHENOMENA}

$\theta_{\mathrm{E}}$ have phases which add and subtract to $\theta_{1}(z)$ and $\theta_{2}(z)$ in such a way as to impose this final condition, $\theta=n 2 \pi$ at $z=\infty$.

One must be careful to correct equation 26, applicable only for $\alpha l<1$, as $N$ increases for a given sample length $l$ to account for the $z$-dependence of the echo. As the effective sample length is increased, because the number of Beer's lengths $\alpha l$ increases, the echo area is modified as a function of pulse propagation distance in the sample, because of the area theorem expressed by equation 28 . Computer plots show for $\log _{10} \theta_{\mathrm{E}}^{2}$ plotted versus $\log _{10} \alpha z$ that $\theta_{\mathrm{E}}^{2}$ reaches a peak and turns over, showing deviation from the $\theta_{\mathrm{E}}^{2} \sim N^{2}$ dependence law. The equations $\mathrm{d} \theta_{1}(z) / \mathrm{d} z=-(\alpha / 2) \sin \theta_{1}(z)$ and $\mathrm{d} \theta_{2} / \mathrm{d} z=-$ $\left(\alpha / 2 \cos \theta_{1}(z) \sin \theta_{2}(z)\right.$ are coupled ${ }^{7}$ to equation 28. The effective $\alpha$ for the second pulse $\theta_{2}$ becomes $\alpha \cos \theta$ because $^{29}$ of the partial saturation imposed by pulse $\theta_{1}$, which does not overlap with $\theta_{2}$. For small echo areas $\theta_{\mathrm{E}}$ a reasonably good evaluation of the photon echo is obtained from the equation

$$
\begin{aligned}
\frac{\mathrm{d} \theta_{\mathrm{E}}}{\mathrm{d} z} & =\frac{\alpha}{2} \sin \theta_{1}\left[1-\cos \theta_{2}\right] \\
& -\frac{\alpha}{2} \sin \theta_{\mathrm{E}} \cos \theta_{1} \cos \theta_{2}, \text { where } \sin \theta_{\mathrm{E}} \sim \theta_{\mathrm{E}}
\end{aligned}
$$

Therefore for small input areas and small output echo, equation 30 yields

$$
\theta_{\mathrm{E}}=\frac{\theta_{1}(0) \theta_{2}^{2}(0)}{2} \theta^{-2 q} \sinh q
$$

where $q=\alpha z / 2$. Note in equation 30 that the echo pulse sees an effective $\alpha$ given by $\alpha \cos \theta_{1} \cos \theta_{2}$. For small areas $\theta$ in general, it is known from previous observations and computer plots that the pulse width $\tau$ varies little with $z$ and retains the same order of size as the input $\tau$. Therefore it is acceptable to consider $\theta_{\mathrm{E}}^{2}$ as proportional to the echo intensity. Also for small $\theta_{1}(0), \theta_{2}(0)$, only a single photon echo appears which has a measurable intensity, and subsequent echoes are negligible.

It is significant that the peaking of echo intensity $\theta_{\mathrm{E}}^{2}$, occurring at $\alpha z=\ln 3$, shows that the $\alpha$-value at which the turnover occurs $\left(\mathrm{d} \theta_{\mathrm{E}} / \mathrm{d} z=0\right)$ is rather insensitive to various initial values of $\theta_{1}(0), \theta_{2}(0)$, for $\theta_{1}(0), \theta_{2}(0)<\pi$. Under these conditions it can be shown that echo $T_{2}^{\prime}$ damping does not affect this conclusion.

\section{REFERENCES}

1 R. H. Dicke, Phys. Rev. 93, 99 (1954).

2 R. P. Feynman, F. L. Vernon, Jr. and R. W. Hellwarth, J. Appl. Phys. 28, 49 (1957).

3 M. Scully et al., Proceedings of the Physics of Quantum Electronics, Vols. I-IV. Flagstaff, Arizona (1968-1969).

4 M. Haken, Handbuch der Physik, Vol. XXV/2C, Edited by S. Flügge. Springer-Verlag, Freiburg.

5 I. D. Abella, N. A. Kurnit, and S. R. Hartmann, Phys. Rev. 141, 391 (1966).

6 S. L. McCall and E. L. Hahn, Phys. Rev. 183, 457 (1969).

7 S. L. McCall and E. L. Hahn, Phys. Rev. A2, 861 (1970).

8 S. L. McCall and E. L. Hahn (to be published).

9 N. Bloembergan and R. V. Pound, Phys. Rev. 76, 1059 (1949). 


\section{E. L. HAHN}

${ }^{10}$ A procedure used to obtain the area theorem is in ref. 6 .

11 F. T. Arecchi and E. Courtens, Phys. Rev. A2, 1730 (1970).

12 E. Courtens, Phys. Rev. Letters 21, 3(1968).

13 A. Compaan and I. D. Abella, Phys. Rev. Letters 27, 23 (1971).

${ }^{14}$ E. L. Hahn, N. S. Shiren and S. L. McCall, Phys. Letters 37A, 265 (1971). 